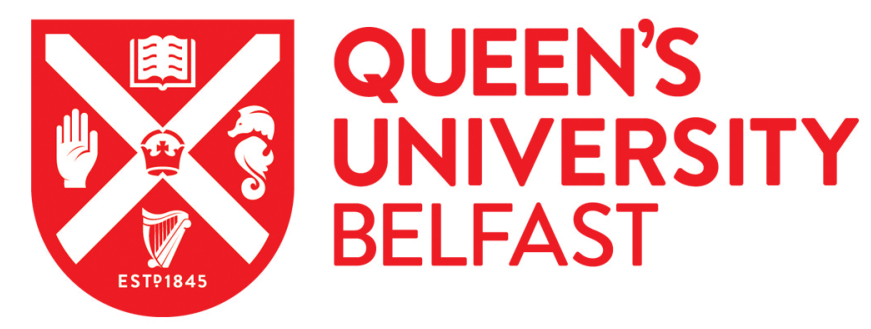

\title{
Laboratory formation of a scaled protostellar jet by coaligned poloidal magnetic field
}

\author{
Abertazzi, R., Ciardi, A., Nakatsutsumi, M., Vinci, T., Beard, J., Bonito, R., Billette, J., Borghesi, M., Burkley, Z., \\ Chen, S. N., Cowan, T. E., Herrmannsdoerfer, T., Higginson, D. P., Kroll, F., Pikuz, S. A., Naughton, K., \\ Romagnani, L., Riconda, C., Revet, G., ... Fuchs, J. (2014). Laboratory formation of a scaled protostellar jet by \\ coaligned poloidal magnetic field. Science, 346(6207), 325-328. https://doi.org/10.1126/science.1259694
}

\section{Published in:}

Science

\section{Document Version:}

Peer reviewed version

Queen's University Belfast - Research Portal:

Link to publication record in Queen's University Belfast Research Portal

\section{Publisher rights}

Copyright 2014 the authors.

This is the author's version of the work. It is posted here by permission of the AAAS for personal use, not for redistribution. The definitive version was published in Science, volume 346, issue 6207, DOI: 10.1126/science.1259694.

\section{General rights}

Copyright for the publications made accessible via the Queen's University Belfast Research Portal is retained by the author(s) and / or other copyright owners and it is a condition of accessing these publications that users recognise and abide by the legal requirements associated with these rights.

Take down policy

The Research Portal is Queen's institutional repository that provides access to Queen's research output. Every effort has been made to ensure that content in the Research Portal does not infringe any person's rights, or applicable UK laws. If you discover content in the Research Portal that you believe breaches copyright or violates any law, please contact openaccess@qub.ac.uk. 


\title{
Laboratory formation of a scaled protostellar jet by co-aligned poloidal magnetic field
}

Authors: B. Albertazzi ${ }^{1,2}$, A. Ciardi ${ }^{3}$, M. Nakatsutsumi ${ }^{1}$, T. Vinci ${ }^{1}$, J. Béard ${ }^{4}$, R. Bonito ${ }^{5}$, J. Billette $^{4}$, Z. Burkley ${ }^{1}$, S.N. Chen ${ }^{1}$, T. E. Cowan ${ }^{6,7}$, A.Ya. Faenov ${ }^{8,9}$, T. Herrmannsdörfer ${ }^{7}$, D. P. Higginson $^{1}$, F. Kroll ${ }^{6,7}$, S. A. Pikuz ${ }^{8}$, K. Naughton ${ }^{10}$, L. Romagnagni ${ }^{1}$, C. Riconda ${ }^{1}$, G. Revet ${ }^{1}$, R. Riquier $^{1,11}$, H-P. Schlenvoigt ${ }^{6,7}$, I.Yu. Skobelev ${ }^{8}$, A. Soloviev ${ }^{12}$, M. Huarte-Espinosa ${ }^{13}$, A. Frank $^{13}$, O. Portugall ${ }^{4}$, H. Pépin ${ }^{2}$, J. Fuchs ${ }^{1,11, *}$

\section{Affiliations:}

${ }^{1}$ LULI, École Polytechnique, CNRS, CEA, UPMC, 91128 Palaiseau, France.

${ }^{2}$ INRS-EMT, Varennes, Québec, Canada.

${ }^{3}$ LERMA, Observatoire de Paris, CNRS UMR 8112, Paris France.

${ }^{4}$ LNCMI, UPR 3228, CNRS-UFJ-UPS-INSA, 31400 Toulouse, France.

${ }^{5}$ Dipartimento di Fisica e Chimica Università di Palermo - INAF - Osservatorio Astronomico di Palermo, Italy •

${ }^{6}$ Technische Universität Dresden, 01062 Dresden, Germany.

${ }^{7}$ Helmholtz-Zentrum Dresden-Rossendorf, Bautzner Landstr. 400, 01328, Dresden, Germany.

${ }^{8}$ Joint Institute for High Temperatures, RAS, 125412, Moscow, Russia

${ }^{9}$ Photon Pioneers Center, Osaka University, Osaka 565-0871, Japan

${ }^{10}$ Department of Physics and Astronomy, Queen's University Belfast, Belfast BT7 1NN, UK

${ }^{11}$ CEA, DAM, DIF, 91297 Arpajon, France

${ }^{12}$ Institute of Applied Physics, 46 Ulyanov Str., 603950 Nizhny Novgorod, Russia

${ }^{13}$ Department of Physics and Astronomy, University of Rochester, Rochester NY, USA.

*Correspondence to: julien.fuchs@ polytechnique.fr.

\begin{abstract}
:
Although narrow bipolar jets of matter have been observed to emerge from a wide variety of astrophysical systems, the question of their formation and morphology, past their launching by magneto-centrifugal forces, is still open. Our scaled laboratory experiments, representative of outflows from young stellar objects (YSOs), reveal that stable collimation of the entire flow into a narrow jet can result from the presence of a large-scale poloidal magnetic field that is consistent with observations. The observed plasma flow focuses and creates an interior cavity, giving rise, close to the source, to a standing conical shock from which the jet emerges. Together with astrophysical full-scale simulations, we conclude that this can also explain recently discovered X-ray emission features observed from low-density regions at the base of protostellar jets, such as the well-studied jet HH 154.
\end{abstract}




\section{One Sentence Summary:}

Laboratory experiments reveal that the shaping of narrow jets emerging from young stars can be explained by axial magnetic fields in which the stars are embedded.

\section{Main Text:}

Bipolar narrow jets are not only spectacular matter ejecta commonly observed in the universe to stream along the axis of rotation of varied objects, such as young stellar objects (YSOs) surrounded by an accretion disk, but are also thought to play a key role in their evolution. Hence, understanding their formation is key to understand mass, energy and angular momentum redistribution between the dense core and the parent cloud. Once formed, it is understood that these jets can continue ballistically over large distances. However, how does the narrow jet form and what are its characteristics? The accepted standard model $(1,2)$ of matter extraction and launching involves poloidal magnetic field anchored in the disk. Here, poloidal is referred to as broadly speaking axial with respect to the jet flow, in opposition to the toroidal component in cylindrical geometry that winds around the jet. In the model, matter is magnetocentrifugally accelerated into wide-angle conical winds (3). As the magnetic field lines become twisted by the inertia of the initially co-rotating plasma, the toroidal component of the magnetic field leads to self-collimation of the wind. Nonetheless, this process cannot solely and satisfactorily explain collimation of the flow into narrow jet-like form. First, self-collimation cannot account for the confinement of the whole outflow structure. Indeed, the winds in these models formally extend to infinity and have difficulties accounting for the observed jet widths (4). Furthermore, jets dominated by a toroidal magnetic field are prone to current and pressuredriven instabilities which can disrupt the jet (5). Efforts to improve the model have led to studies of truncated disk winds where, in order to collimate the flow, the whole outflow structure has to rely on the thermal pressure of a surrounding medium (6), the presence of which has of yet to be established.

Alternatively, outflow confinement by a large-scale ordered poloidal magnetic field (7) has been explored in numerical simulations (8). Although evidence for such fields near the jet source is mostly indirect so far, it should be noted that they are well established on larger scales (9), and observed to be aligned (within $~ 35$ degrees) with the bipolar outflow axes of young star objects (YSO). These earlier numerical studies showed that such poloidal field could convert ejected matter from weakly collimated to parallel streamlined, although the simulations lacked a proper treatment of plasma cooling and were constrained by computing limitations to simulate the flow over only small distances from the source. More recently, we revisited the poloidal confinement scenario using large-scale, high resolution 3D MHD simulations (10), which included cooling, and showed that the whole outflow could be constrained. Notably, we predicted a particular morphology for the outflow with a shock-bounded cavity followed by a narrow jet. We note here that such mechanism does not exclude magneto-centrifugal selfcollimation: even if poloidal collimation can act on its own, it can also be complementary to selfcollimation by stabilizing and collimating further the jets produced by the latter. 
We report here on scaled laboratory experiments exploiting unprecedented coupling of high-amplitude and large-scale magnetic field to high-velocity plasma flows so as to create conditions representative of a YSO. Our measurements conclusively demonstrate that stable jets can indeed be collimated from a wide-angle star/disk wind embedded in co-aligned, large-scale poloidal magnetic field. This is notably obtained without having to rely on the collimation by an external medium. We also show that stable collimation of the whole flow into a narrow jet can be obtained even in the case of an angular offset (up to $40^{\circ}$ ) between the magnetic field and the outflow axis. The transition from a divergent to a narrowly collimated flow results from the plasma being redirected toward the axis by a shock structure induced by the compression of the magnetic field lines under the action of the expanding plasma. As a consequence, this results in a standing conical shock feature at the converging point where the plasma becomes reheated. Full scale astrophysical simulations of an isotropic wind embedded in a large scale magnetic field performed under conditions typical of YSOs are consistent with the laboratory observations. By post processing the astrophysical simulations, we determine that the shock focused region generates an X-ray source with luminosity and location in agreement with the Chandra

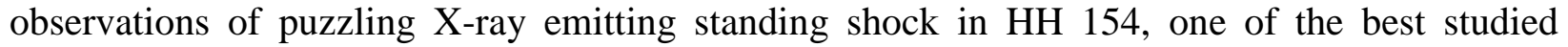
astrophysical jet emerging from a YSO (11).

The laboratory plasma, mimicking a YSO flow, is produced by short $(0.5 \mathrm{~ns})$, high-power laser irradiation of massive solid (plastic) targets. The plasma is a highly conductive and superfast-magnetosonic flow. It expands into a steady ( $>5 \mu \mathrm{s})$ and homogeneous axial magnetic field extending over a large volume $(3 \mathrm{~cm}$ longitudinally by $1 \mathrm{~cm}$ diameter radially) with strength of up to $0.4 \mathrm{MG}$ (see Ref. 12 and references therein). The plasma is thermally launched from a region on the target on the order of the laser spot diameter $(0.75 \mathrm{~mm})$ and this wide-angle flow is, as in a YSO, initially dominated by its kinetic energy. It is well approximated by ideal magnetohydrodynamics (13) ensuring its relevance as a scaled astrophysical plasma. Indeed, the dimensionless Reynolds (Re), magnetic Reynolds (ReM), and Peclet (Pe) numbers are much larger than unity $\left(\operatorname{Re} \sim 10^{4}-10^{5}\right.$; ReM $\left.\sim 50 ; \mathrm{Pe} \sim 3-5\right)$. This implies that the advective transport of momentum, magnetic field, and thermal energy dominates over diffusive transport as expected in a YSO outflow. The scaling between the laboratory plasma and a YSO outflow allows also us to scale a time span of $20 \mathrm{~ns}$ in the laboratory to an equivalent $\sim 6 \mathrm{yrs}$ in the natural environment, during which it propagates over $\sim 600 \mathrm{AU}$. Hence, even over short time scales $(<100 \mathrm{~ns})$, the experiment has the ability to sample the stationary morphology of an astrophysical outflow. The strength of the magnetic field in which the plasma is embedded is much larger than in the astrophysical environment ( $\mathrm{mG}$, see Ref. 14) in order to compensate the shortness of the space and time scales of the experiment. This means that the ratio of the plasma kinetic ram pressure versus the magnetic pressure will transit from $>1$ to $<<1$ over a few $\mathrm{mm}$, while it does the same over a few tens of AU in a YSO. Finally, we note that toroidal magnetic fields, which are selfgenerated in the plasma outflow due to crossed density and temperature gradients (15), are in our case of low amplitude due to the low laser intensity employed here (see Methods) and at the same time limited to zones close $(<0.5 \mathrm{~mm})$ to the target surface and hence are not able to confine the flow past this point, as can be observed from Fig $1 \mathrm{C}$ where, without external field, the plasma is seen to expand at wide angle. In short, this experimental configuration mimics well the propagation into a poloidal magnetic field of one hemisphere of a naturally occurring YSO spherical wind; the latter emerging at large distances from the disk, where acceleration is complete and gravitational effects are unimportant. 


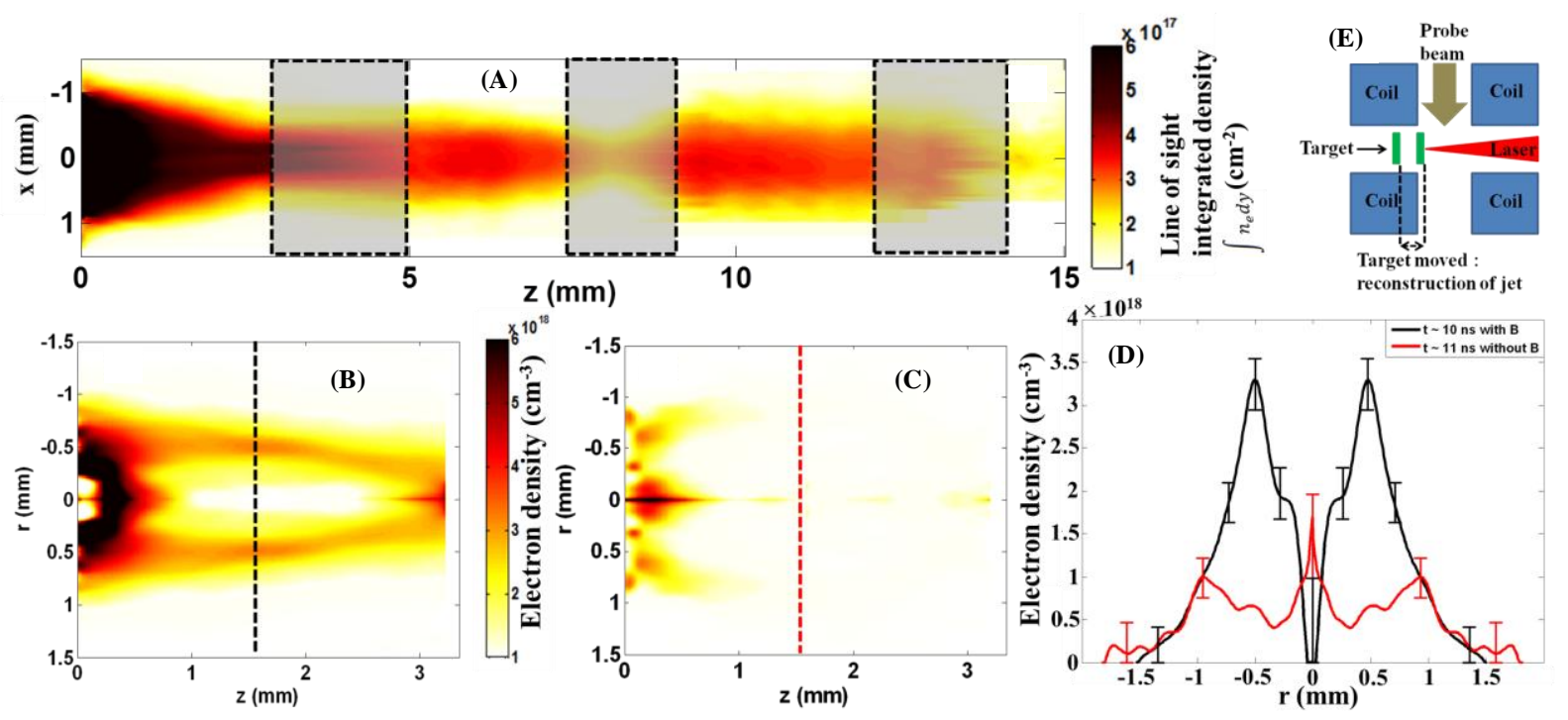

Fig. 1. Laboratory demonstration of jet formation by axial magnetic field. (A) Plasma integrated density measured $20 \mathrm{~ns}$ after the laser irradiation (coming from the right) of a $\mathrm{CH}$ target (left) immersed in the z-oriented 0.2 MG magnetic field. Four spatially patched images are used (see E). Shaded areas are linear interpolation in between observed sections of the jet. (B-C) Abel inverted density maps in the case with (B) and without (C) magnetic field, and (D) lineouts (along the dashed lines shown in B and C), show the cavity region, and plasma convergence on axis, induced by the magnetic field. The error bars on (D) represent the difference in the plasma density retrieved from the upper and lower measured phase maps (see Methods).

The typical morphology of such laboratory plasma measured at $20 \mathrm{~ns}$ after the initiation of the plasma expansion in the presence of a $0.2 \mathrm{MG}$ axial magnetic field, is shown in Fig 1A. A measurement of the electron areal density of the expanding plasma obtained through transverse optical laser probing is presented. The axial magnetic field is seen to have a profound effect on the collimation of the laser-produced plasma flow, thus leading to the appearance of a narrow jet with an aspect ratio of $>10$ that is maintained over the entire homogeneous magnetic field region with little variation in density. Such tight collimation is also observed when imaging the x-ray emission in $\mathrm{keV}$ range originating from the plasma, and is in excellent agreement with our earlier MHD numerical investigations (10). We emphasize that even laser experiments specifically designed to produce jets from radiatively cooled, unmagnetized, plasmas do not exhibit such morphology, and have in fact much lower aspect ratios $(16,17,18)$. The mechanism leading to the tight magnetic collimation of the plasma plume is illustrated in Fig $1 \mathrm{~B}$ and $1 \mathrm{C}$, which show Abel inverted interferometric images of the base of the flow with and without the magnetic field. It is further illustrated in Fig 2 by three-dimensional resistive MHD numerical simulations using the parameters of the experiment (see Methods). When the plasma expands in the presence of a magnetic field (Fig 1B), one clearly observes in the experiment the formation of a converging cavity having an outer shell of higher electron density. We stress that neither the cavity, nor the jet are observed in the case when no magnetic field is applied (see Fig 1C). The formation of this shell is the consequence of the piling up and heating of plasma in a fast-mode oblique shock generated by the external magnetic field halting the radial expansion of the flow. Since the 
plasma is of high temperature and has a super-fast-magnetosonic expansion speed ( $v \sim 200-500$ $\mathrm{km} / \mathrm{s}$, similar to the outflow velocity measured in YSO), the magnetic field lines are bent and compressed past this shocked envelope, as shown in Fig 2.

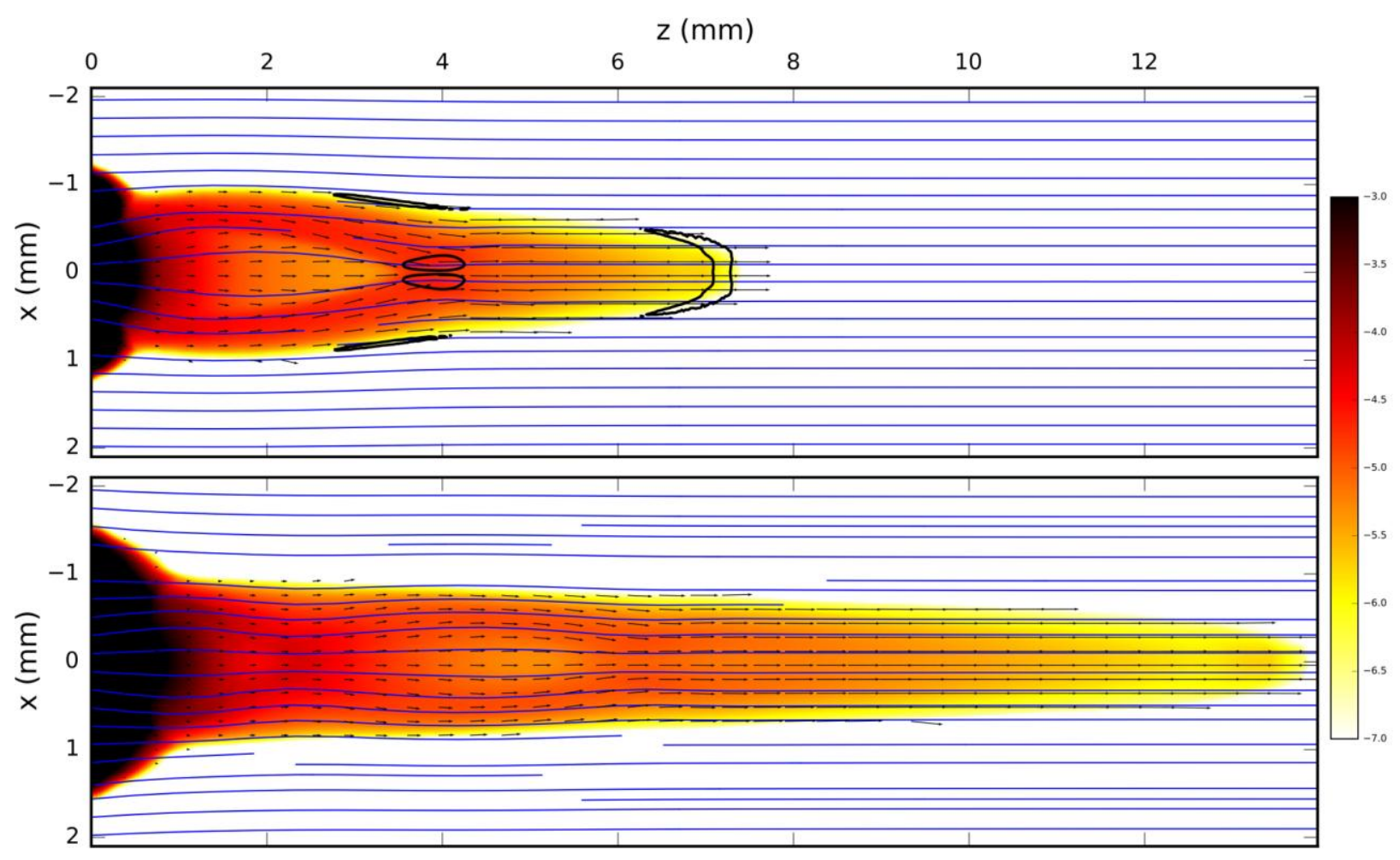

Fig. 2. 3D MHD modelling of the experiment, illustrating the formation of a heated steady shock region at the tip of the cavity and subsequent jet collimation. The maps show two snapshots ((A) $10 \mathrm{~ns}$ and (B) $20 \mathrm{~ns}$ from the end of the laser pulse) of the density $\left(\log _{10} \rho\right.$ in g. $\mathrm{cm}^{-3}$ ) along X-Z. The arrows represent velocity vectors and the lines magnetic field. At $10 \mathrm{ns,}$ the plasma can be seen sliding along the compressed magnetic field and converging on axis, forming a shock $(\mathrm{z} \sim 4 \mathrm{~mm})$. The black contour line shows plasma heating above $70 \mathrm{eV}$.

The expanding plasma from the target is refracted across this oblique shock and slides along the walls of the cavity which have been curved toward the axis by the magnetic forces. When the flow reaches a convergence point it stagnates, forming a conical shock. It is this shock that focuses the flow in the axial direction and generates a narrow jet ahead the convergence point. This convergence of plasma towards the axis $(z \sim 3 \mathrm{~mm})$ is visible in the experimental images (Fig 1A and 1B), and simulation reveals that the plasma becomes heated to $\sim 70 \mathrm{eV}$ by this shock. When applying the external magnetic field, significant plasma heating, compared to what is observed in the freely expanding plasma, is also seen by our spectrally-resolved x-ray emissivity diagnostic, consistently with earlier experiments (19). Interestingly, this mechanism of jet formation is similar to astrophysical models of hydrodynamic collimation of a wind by the inertia of a dense, torus-like circumstellar envelope (20). However, it is demonstrated here that it can clearly work even in the absence of a surrounding medium. 
The overall jet formation process illustrated here for a particular set of laser and target conditions was observed to be very repeatable and effective over a wide variety of different experimental conditions, and was always found in agreement with MHD modeling, hence validating the physical mechanism described above. Notably, when the laser intensity on target was increased, we could increase the kinetic pressure at the target surface. This produced a wider cavity and moved the shock convergence region further away along the jet axis. Similarly, we could also move the distance of the shock with respect to the plasma source by varying the magnetic field strength. We note that, contrary to what takes place in the experiment, if the deposition of energy would be continuous, the location of the shock convergence region of the jet would be stationary. Finally, when we tilted the target and so the axis of the plasma expansion with respect to the magnetic field axis, we could still observe the cavity formation and plasma focusing on axis, even for angular offset up to $40^{\circ}$, showing that the mechanism is robust.

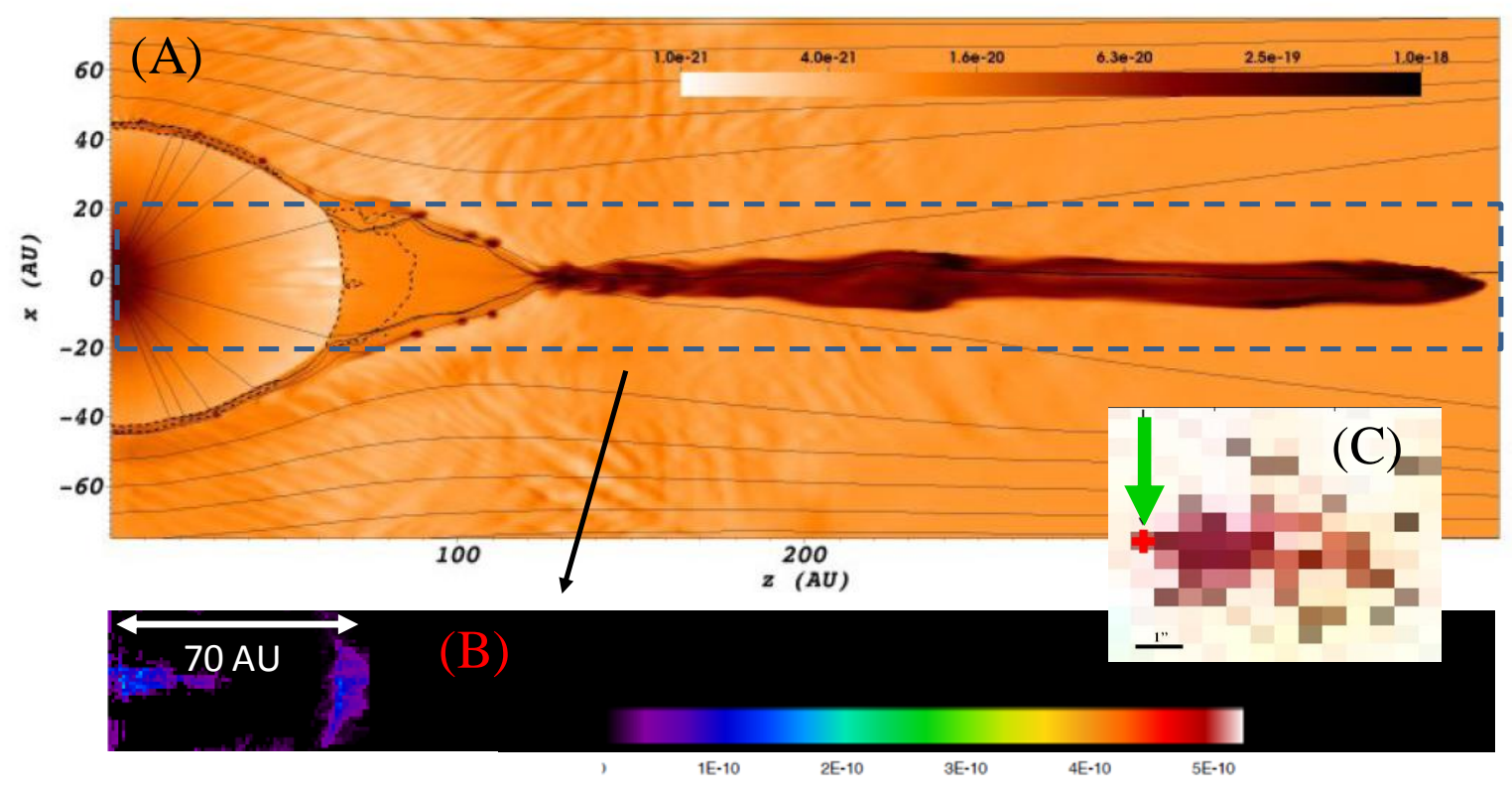

Fig. 3. 3D simulation of jet formation and collimation in a young star system embedded in a 5 mG axial magnetic field. An isotropic star/disk wind with a mass ejection rate of $10^{-8} \mathrm{M}_{\odot} /$ year and velocity $200 \mathrm{~km} / \mathrm{s}$ is embedded in an initially axial (z) magnetic field. (A) (x-z) mass density $\left(\log _{10} \rho\right.$ in $\left.\mathrm{g} . \mathrm{cm}^{-3}\right)$ at time 20 years. Black lines: magnetic field lines; dashed contour: plasma of temperature $\geq 70 \mathrm{eV}$. (B) post-processed (21) X-ray emission of (A) (counts/s in each pixel with size of $1 \mathrm{AU})$. (C) X-ray image of HH154 measured by the Chandra telescope. The green arrow points to the star.

As shown in Fig 3A, the same morphology is observed in full-scale astrophysical simulations of an isotropic star/disk wind embedded in a large scale magnetic field (see Methods), performed with mass-ejection rates (22) and magnetic field strengths (14) typical of YSO. Supporting the scalability of laboratory experiment, we observe in the simulation the essential dynamics and features observed in the laboratory flows. We performed extensive variations of wind and field parameters, and verified with detailed numerical simulations that the poloidal field induced jet collimation mechanism was a very robust process. 
An interesting outcome of our study is that typical YSO should then have a stationary region of shock heated plasma that forms within a few tens to one hundred AU from the wind source (see Fig 3A). This numerical prediction can be directly compared to the analysis $(23,24,25)$ of observations made over more than one decade using X-ray satellites that have revealed (see Fig 3C) bright sources of stationary X-ray emission zones located at the base of jets ( 100 AU from the source) emerging from YSO. They have been, hitherto, unexplained using self-collimation models of jet formation, but are however well consistent with the process revealed here. Indeed, post-processing (see Fig 3B) of astrophysical simulation shows that the shock-focused region at the tip of the cavity has a luminosity and a distance to the source that is consistent with the stationary X-ray emission feature located close ( 60-80 AU) to the base of one of the nearest and most luminous jet observed in the X-ray band, namely HH154.

Beyond proposing a new, simple and plausible scenario for the collimation of narrow stable jet past its launching phase $(1,2)$, consistent with recent astrophysical observations $(9,24,25)$, and helping to advance the understanding of jet/core interaction in YSO, our work opens the possibility to study and/or model important aspects of jet physics in the laboratory. These include, e.g., transverse instabilities that can affect the jet structure, or episodic ejections, i.e. multi-component and time-dependent interacting winds, which can be easily simulated in the laboratory by using multiple laser pulses separated by a few ns. Producing such magnetized narrow plasma columns and letting them impact a solid will also uniquely allow to study plasma dynamics in accretion columns in young stars, i.e. model magnetic arches that are loaded with disk material which free-falls towards the star. Beyond these aspects, adapting the present experimental work to other configurations will permit advances in resolving pending questions regarding a wide range of astrophysical and plasma physics systems where magnetic fields are thought to play a significant role.

Acknowledgments: We acknowledge the support of the LULI teams, and discussions with P. Audebert. This work was supported by Grant No. E1127 from Région Ile-de-France, ANR Blanc Grant $\mathrm{n}^{\circ}$ 12-BS09-025-01 SILAMPA, Grant ELAM from RTRA-Saclay and by NSERC Discovery Grant $N^{\circ}$ 26558-2007 RGPIN from Canada. S. N. Chen acknowledges the support received during this work by the National Science Foundation, Grant No. 1064468. This work was supported in part by the Ministry of Education and Science of the Russian Federation under Contract No.14.Z50.31.0007.

J.F. and A.C. designed the project, J.F., H.P., and O.P. designed the experiment, J.Bé., J.Bi., O.P, T.E.C. and T.H. designed the pulsed high-magnetic field system, B.A., M.N., J.F. J.Bé., F.K, S.N.C., H.-P. S., and L.R. performed the experiment, B.A., M.N., T.V., Z.B., and J.F. analyzed the data, A.C., T.V., C.R., M. H.-E., R.B. designed and performed the simulations. J.F., A.C., B.A., H.P. wrote the bulk of the article. T.V., S.N.C, M. H.-E., R.B., and A.F. contributed to the article with substantial edits. All authors provided comments on the various stages of the article.

\section{Supplementary Materials:}

\section{Materials and Methods:}

Magnetic field generation. The generation of the external magnetic field of up to $0.4 \mathrm{MG}$ amplitude, in a non-destructive and repeatable manner compatible with a high-energy laser environment, is done by coupling a $16 \mathrm{kV}$ pulsed energy supply to a split coil (12). The generator 
is composed of five capacitors of $16 \mathrm{kV}, 50 \mu \mathrm{F}$ with a total capacitance of $250 \mu \mathrm{F}$ and a maximum stored energy of $32 \mathrm{~kJ}$. The energy can be released over at $25 \mathrm{kV} / 250 \mathrm{kA}$ ignitron switch to a downstream circuit. It is then connected through a coaxial cable to a resistive splitpair magnet, with each magnet being of $6 \mathrm{~mm}$ inner and 50 to $60 \mathrm{~mm}$ outer diameter. The two coils composing the split assembly are connected in parallel and are separated by a midplane plate in stainless steel, insulated with glass fiber-epoxy sheets that are designed to contain the magnetic pressure. The distance between the two coils is $8 \mathrm{~mm}$, which is a compromise between electrical insulation, mechanical strength of the midplane plate and the possibility to produce safely up to $40 \mathrm{~T}$ ( $20 \mathrm{~T}$ was used in Fig 1 ) in a volume of $2250 \mathrm{~mm}^{3}$. The number of turns for each coil is 80 . Two tubes of $5 \mathrm{~mm}$ diameter were inserted in the coil, one along the field axis, for the laser beam to enter the interaction region, and the other perpendicular, for diagnosing the plasma plume. While there is vacuum inside the tubes, the split coils remain at air to avoid problems of heat dissipation management and concerns of electrical arcing in moderate vacuum.

Laser experiment. The experiment was performed using the LULI ELFIE laser facility at École Polytechnique, using the non compressed part of the Nd:glass laser beam to create the plasma plume. The laser can deliver up to $50 \mathrm{~J}$ in approximately $500 \mathrm{ps}$ FWHM at the fundamental wavelength of $1.053 \mu \mathrm{m}$. A long focal lens of $2.2 \mathrm{~m}$ served to focus the laser beam at the centre of the split coil in order to immerse the plasma in the zone of highest amplitude and spatially homogeneous magnetic field. In order to vary the kinetic pressure of the plasma at the target surface (composed of $\mathrm{CH}_{2}$, mostly, or $\mathrm{CF}_{2}$, see below), we varied the laser power density from $10^{12} \mathrm{~W} \cdot \mathrm{cm}^{-2}$ (as used in Fig 1) to $10^{14} \mathrm{~W} \cdot \mathrm{cm}^{-2}$. The expanding plasma was probed through the use of an optical beam of few hundred $\mathrm{mJ}, 400 \mathrm{fs}$ duration, frequency-doubled to $527 \mathrm{~nm}$, coupled to a Mach-Zehnder interferometer to quantitatively retrieve the plasma electron density of the expanding plume. The delay between the main laser beam and the probe beam was varied to follow the evolution in time of the plasma plume. To reconstruct the overall jet longitudinal profile, we spatially patched (as in Fig 1A) images since our transverse diagnostic has a limited field of view (see Fig 1E), with the target being moved back for each shot in order to keep the plasma outflow in the homogeneous magnetic field region. To produce the density maps of Fig 1B and 1C, Abel inversion was applied to the phase map measured by interferometry. Since Abel inversion relies on the hypothesis of the plasma being cylindrically symmetric around its expansion axis, we inverted separately the upper and lower phase maps of Fig 1A, and then computed the maximum difference of densities obtained from each panel. The measured difference at each location results in the error bars displayed in Fig 1D (which shows lineouts of Fig $1 \mathrm{~B}$ and $1 \mathrm{C}$ along the dashed lines displayed in each figure). X-ray emission of the jets produced from $\mathrm{CF}_{2}$ targets was also measured by means of focusing spectrometers with $2 \mathrm{D}$ spatial resolution. Besides the mapping of the x-ray source geometry, the relative intensities of spectral lines $(\mathrm{He} \beta, \mathrm{He} \gamma, \mathrm{He} \delta)$ radiated by He-like $\mathrm{F}$ ions were analyzed. As the laser-produced plasma expands beyond $\mathrm{z}>0.5 \mathrm{~mm}$ from the target surface, the spectral line intensities are defined mainly by the recombination processes. In this case the relative intensities of the lines depend only on the electron plasma density $\mathrm{n}_{\mathrm{e}}$ and temperature $\mathrm{T}_{\mathrm{e}}$. Comparing the experimental spectra with the results of quasi-steady radiative-collisional kinetic calculations, the profiles of $\mathrm{T}_{\mathrm{e}}$ along the jet axis could be inferred in the region $\mathrm{z}=0.5-5 \mathrm{~mm}$ from the target surface.

\section{Laboratory Simulations.}


The initial laser target interaction is modeled with the two-dimensional (2D), three-temperature, Lagrangian, radiation hydrodynamic code DUED (26), coupled with SESAME EOS tables (27). The propagation of the plasma in the magnetic field is modeled with the three-dimensional (3D), Eulerian, resistive magnetohydrodynamic code GORGON (28). The regular, cubic-cells grid was initialized with the interpolated profiles of density, temperature (electronic and ionic), and momentum taken from the DUED simulations at the end of the laser pulse (1.2 ns). Simulations were run at different resolutions $(\Delta x=35-65$ microns), showing no significant differences in the results. In addition, to remove the cylindrical symmetry imposed by remapping $2 \mathrm{D}$ axisymmetric simulations onto a 3D grid, a series of simulations were initialized with a random velocity perturbation superimposed on the local initial velocity (5 - 15\%). The results are qualitatively similar to the unperturbed cases, with small differences in the azimuthal structure of the flow.

\section{Astrophysical Simulations.}

The simulations were performed with the adaptive mesh, 3D ideal MHD code RAMSES (29), including cooling processes (30). The magnetic field is evolved using the constrained transport method, preserving the nullity of the divergence of the magnetic field. The simulations were performed using the HLLD solver. The computational domain is initiated with a uniform magnetic field along the z-direction and an isotropic wind injected within a spherical shell with inner and outer radii of 8 and 10 AU respectively. More realistic winds with finite opening angles are more easily recollimated by the magnetic field, thus the simulations with an isotropic wind case represent an extreme (idealized) scenario for testing the effectiveness of the poloidal collimation mechanism. We note that we also explored more realistic field configurations where the field lines diverge with distance above the disk. In this case, the same outflow structure and collimation is achieved compared to the case where the magnetic field is perfectly vertical. The central region of the grid, within a radius of $8 \mathrm{AU}$ is initialized with a constant density and zero velocity, providing the internal boundary for an isotropic wind and fixing the magnetic field lines to vertical. The wind is injected over a small radial extent (8-10 AU) to provide a well resolved flow. In this spherical shell the gas is fixed to constant velocity and a density profile decreasing with radius. These are fixed at each time step. The rest of the volume is filled with a uniform density background gas with a density 25 times smaller than the initial wind density. The grid resolution is adaptively refined to capture steep density gradients. Up to 5 levels of refinement are used providing a resolution as low as $0.25 \mathrm{AU}$. The domain size is $512^{3} \mathrm{AU}$. Regarding the post-processing (21) of the simulation shown in Fig 3A, we derive the distribution of emission measure, $\mathrm{EM}(\mathrm{T})$, where EM is the volume integral of the square of the plasma density and $\mathrm{T}$ is the temperature of the emitting plasma, integrating along the line of sight under the assumption of optically thin plasma, to compute the emissivity image shown in Fig 3B. Assuming proper radiative losses and using available spectral emission codes, we obtain the intrinsic luminosity of the X-ray source and we also synthesize the emission map in the X-ray band taking into account

the instrumental response of the X-ray telescopes and typical values of distance and interstellar absorption derived from the observations of X-ray emitting protostellar jets.

\section{References and Notes:}


${ }^{1}$ R. D. Blandford, D. G. Payne, Hydromagnetic flows from accretion discs and the production of radio jets. Monthly Notices of the Royal Astronomical Society 199, 883-903 (1982).

2 J. Ferreira, Magnetically-driven jets from Keplerian accretion discs. Astronomy and Astrophysics 319, 340-359 (1997).

${ }^{3}$ R. E.Ainsworth, , T. P. Ray, A. M. M. Scaife, J. S. Greaves, R. J. Beswick, Subarcsecond highsensitivity measurements of the DG Tau jet with e-MERLIN. Monthly Notices of the Royal Astronomical Society 436, L64-L68 (2013).

${ }^{4}$ M. Stute, J. Gracia, K. Tsinganos, N. Vlahakis, Comparison of synthetic maps from truncated jet-formation models with YSO jet observations. Astronomy and Astrophysics 516, A6 (2010).

${ }_{5}^{5}$ R. Moll, H. C. Spruit, M. Obergaulinger, Kink instabilities in jets from rotating magnetic fields. Astronomy and Astrophysics 492, 621-630 (2008).

${ }^{6}$ T. Matsakos, et al. Two-component jet simulations. II. Combining analytical disk and stellar MHD outflow solutions. Astronomy and Astrophysics 502, 217-229 (2009).

${ }^{7}$ H. C. Spruit, T. Foglizzo, R. Stehle, Collimation of magnetically driven jets from accretion discs. Monthly Notices of the Royal Astronomical Society 288, 333-342 (1997).

${ }^{8}$ S. Matt, R. Winglee, K.-H. Böhm, Collimation of a central wind by a disc-associated magnetic field. Monthly Notices of the Royal Astronomical Society 345, 660-670 (2003).

9 N. L. Chapman, et al. Alignment between flattened protostellar infall envelopes and ambient magnetic fields. The Astrophysical Journal 770, 151 (2013).

10 A. Ciardi A. et al. Astrophysics of Magnetically Collimated Jets Generated from LaserProduced Plasmas. Physical Review Letters 110, 25002 (2013).

$11 \mathrm{~F}$. Favata et al. The discovery of an expanding X-ray source in the HH 154 protostellar jet. Astronomy and Astrophysics 450L, 17 (2006).

12 B. Albertazzi et al. Production of large volume, strongly magnetized laser-produced plasmas by use of pulsed external magnetic fields Rev. Sci. Instrum. 84, 043505 (2013).

${ }^{13}$ D. D. Ryutov, R. P. Drake, B. A. Remington, Criteria for Scaled Laboratory Simulations of Astrophysical MHD Phenomena. The Astrophysical Journal Supplement Series 127, 465-468 (2000).

${ }^{14}$ P. Hartigan, A. Frank, P. Varniére, E. G. Blackman, Magnetic Fields in Stellar Jets. The Astrophysical Journal 661, 910-918 (2007).

15 J. A. Stamper, Review on spontaneous magnetic fields in laser-produced plasmas: Phenomena and measurements. Laser Part. Beams 9, 841-862 (1991).

${ }^{16}$ D. Farley et al. Radiative Jet Experiments of Astrophysical Interest Using Intense Lasers. Phys. Rev. Lett. 83, 1982-1985 (1999).

${ }^{17}$ B. Loupias et al. Supersonic-Jet Experiments Using a High-Energy Laser, Phys. Rev. Lett. 99 (2007) 265001.

${ }^{18} \mathrm{Ph}$. Nicolaï, et al. Studies of supersonic, radiative plasma jet interaction with gases at the Prague Asterix Laser System facility. Phys. Plasmas 15, 082701 (2008)

19 A. Faenov et al. Using X-ray spectroheliograph technique for investigations of laser-produced plasma under interaction with strong magnetic field. Physica Scripta 53, 591-596 (1996)

${ }^{20}$ B. Balick, A. Frank, Shapes and Shaping of Planetary Nebulae. Annual Review of Astronomy and Astrophysics 40, 439-486 (2002).

${ }^{21}$ R. Bonito, S. Orlando, G. Peres, G., F. Favata, F., R. Rosner, The X-ray emission mechanism in the protostellar jet HH 154. Astronomy and Astrophysics 424, L1-L4 (2004) 
22 S. Cabrit, The accretion-ejection connexion in T Tauri stars: jet models vs. observations. in Star-Disk Interaction in Young Stars. Proceedings IAU Symp. 243, (eds Bouvier, J. \& Appenzeller, I.) 203-214 (Cambridge: Cambridge Univ. Press, 2007).

${ }^{23}$ M. Güdel, S. L. Skinner, M. Audard, K. R. Briggs, S. Cabrit, S. Discovery of a bipolar X-ray jet from the T Tauri star DG Tauri. Astronomy and Astrophysics 478, 797-807 (2008).

${ }^{24}$ P. C. Schneider, H. M. Günther, J. H. M. M. Schmitt, The X-ray puzzle of the L1551 IRS 5 jet. Astronomy and Astrophysics 530, A123 (2011).

25 R. Bonito, S. Orlando, M. Miceli, G. Peres, G. Micela, F. Favata, X-Ray emission from protostellar jet HH 154: the first evidence of a diamond shock? The Astrophysical Journal 737, 54 (2011).

${ }^{26} \mathrm{~S}$. Atzeni, et al. Fluid and kinetic simulation of inertial confinement fusion plasmas. Computer Physics Communications 169, 153-159 (2005).

27 See National Technical Information Service Document No. DE94011699, "SESAME database" by J. D. Johnson, Los Alamos National Laboratory Report No. LA-UR-94-1451, 1994. Copies may be ordered from the National Technical Information Service, Springfield, VA 22161.

${ }^{28}$ A. Ciardi, et al. The evolution of magnetic tower jets in the laboratory. Physics of Plasmas 14, 6501 (2007).

29 R. Teyssier, Cosmological hydrodynamics with adaptive mesh refinement. A new high resolution code called RAMSES. Astronomy and Astrophysics 385, 337-364 (2002).

${ }^{30}$ E. Audit, and P. Hennebelle, Thermal condensation in a turbulent atomic hydrogen flow. Astronomy and Astrophysics 433, 1-13 (2005). 